\title{
Robotic redo pancreaticojejunostomy for stenosis following pancreaticoduodenectomy: an alternative technique
}

\author{
Marcel Autran C MACHADO, Fábio F MAKDISSI, Marcel C C MACHADO and José Celso ARDENGH
}

Received: 23 January 2020

Accepted: 7 February 2020

Pancreaticoduodenectomy is the procedure of choice for several diseases from benign or pre-malignant lesions to malignancy ${ }^{(1)}$. One of the most frequent late complications is the stenosis of the pancreato-enteric anastomosis ${ }^{(2-4)}$. This complication is observed in $25 \%-60 \%$ of radiological images during follow-up ${ }^{(2)}$. Although frequent, this stenosis is often asymptomatic but in certain circumstances this stenosis may lead to recurrent acute pancreatitis, pain and pancreatic exocrine insufficiency. About $2 \%$ of all patients will develop a symptomatic pancreaticojejunostomy stricture needing treatment ${ }^{(3,4)}$. We present an alternative technique for the surgical revision of pancreaticojejunostomy using robotic approach. A 60-year-old woman underwent laparoscopic Whipple procedure two years ago. After 6 months, she presented with acute pancreatitis. MRI showed mild dilation of the main pancreatic duct and the patient was conservatively treated. However, since then, she presented several episodes of acute pancreatitis. MRI showed a complete stenosis of the pancreaticojejunostomy with pronounced dilation of the main pancreatic duct (FIGURE 1). Multidisciplinary team decided for revision surgery (E-VIDEO*). Robotic

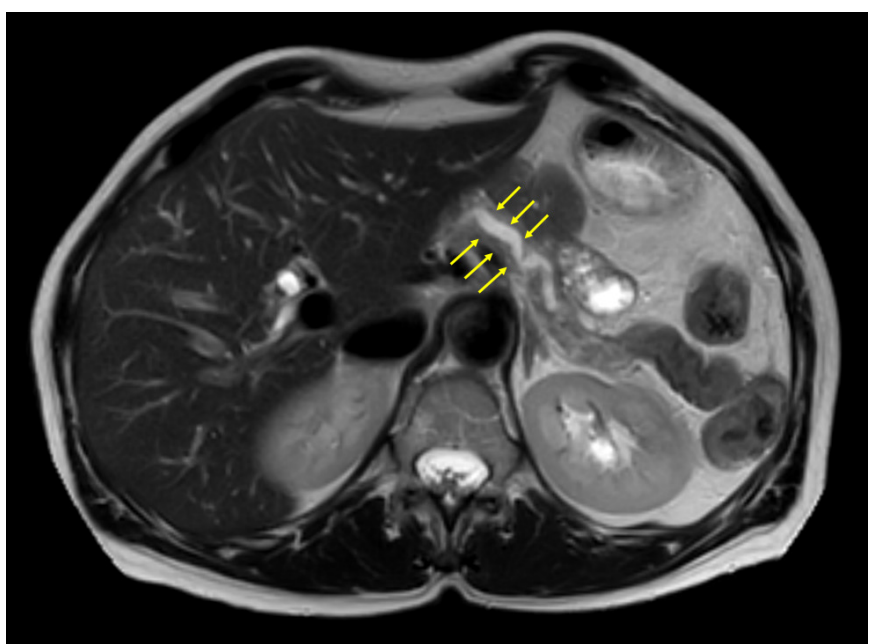

FIGURE 1. Preoperative MRI shows stenosis of the pancreato-jejunostomy with marked dilation of the distal main pancreatic duct. redo pancreaticojejunostomy was indicated (FIGURE 2). A new technique was used (E-VIDEO*). After adhesiolysis, the pancreatoenteric anastomosis is identified (FIGURES 3.A and 3.B). Distal pancreas is then detached from the jejunal loop with scissors from anterior towards posterior interrupted sutures. The duct-tomucosa anastomosis area, which is stenotic is divided with scissors. However, it is completely fibrotic, and the previous anastomosis cannot be identified. The posterior layer is kept intact. Next step is to remove a part of the proximal pancreas in a pyramidal shape towards the posterior layer. This maneuver removes the fibrotic area and the dilated pancreatic duct is opened (FIGURES 3.C and 3.D). A small opening in the jejunum is performed and a ductto-mucosa anastomosis is performed using absorbable continuous suture (FIGURES 4.A and 4.B). Anastomosis is completed with interrupted seromuscular-pancreatic sutures (FIGURES 4.C and 4.D). Operation is completed with drainage of the abdominal

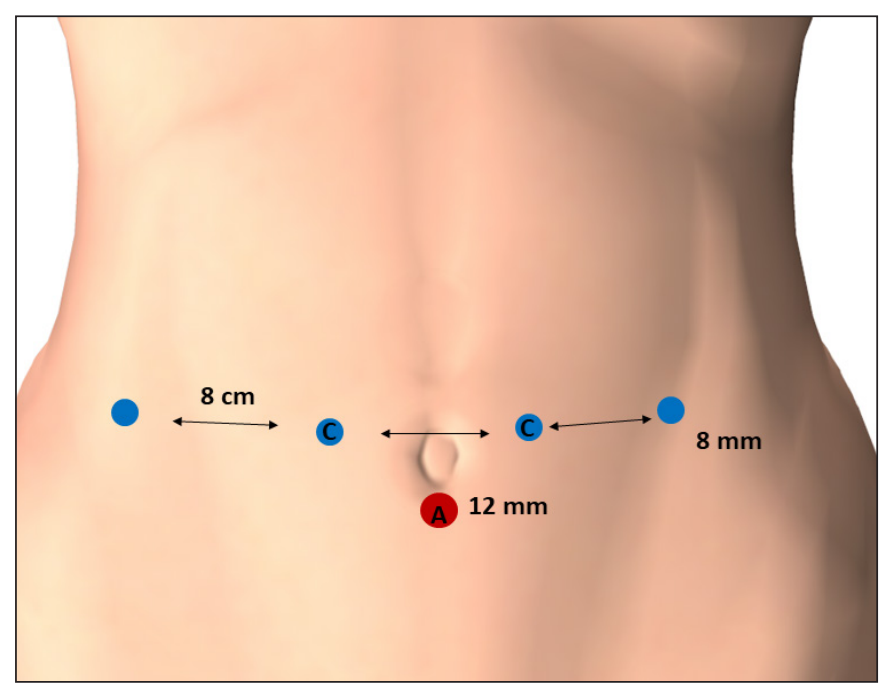

FIGURE 2. Schematic drawing shows type and position of trocars used. The camera $(\mathrm{C})$ is used in two different positions. A. auxiliary port used by bedside surgeon (red dot). A minimum $8 \mathrm{~cm}$ distance should be kept between robotic arms (blue dot).

Declared conflict of interest of all authors: none

Disclosure of funding: no funding received

Nove de Julho Hospital, São Paulo, SP, Brasil.

Corresponding author: Marcel Autran C. Machado, M.D. E-mail: dr@drmarcel.com.br

*Video: https://www.youtube.com/watch?v=WOEOgyYI_xk 


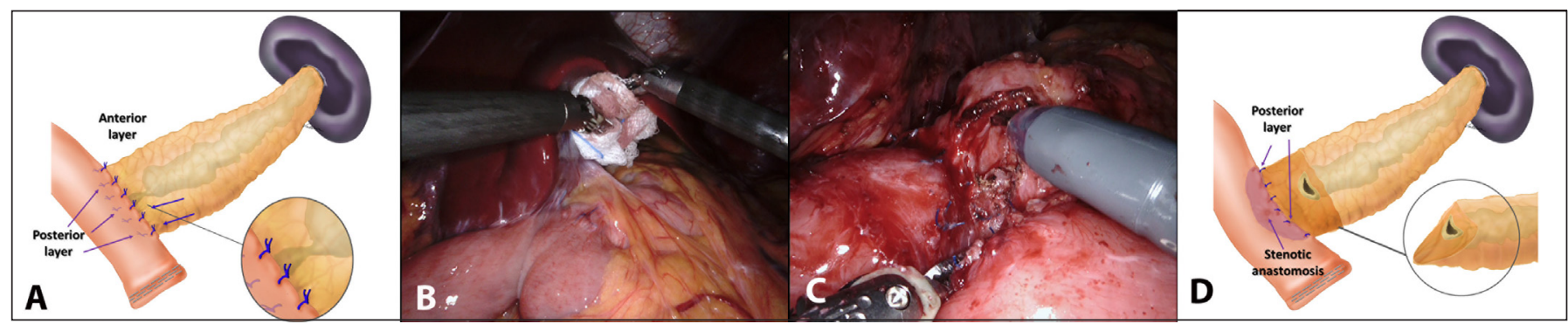

FIGURE 3. Alternative technique for redo pancreaticojejunostomy.

A. Schematic drawing shows pancreaticojejunostomy with stenosis of duct-to-mucosa anastomosis. Close-up shows the site of stenosis. B. Intraoperative view after adhesiolysis. Pancreaticojejunostomy is identified. C. Intraoperative view after removal of the anterior layer and pyramidal resection of the pancreas exposing the dilated main pancreatic duct. Posterior layer is preserved to maintain the anastomosis assembled. D. Schematic drawing after removal of the anterior layer and partial pancreatic resection shows dilated main pancreatic duct, stenotic duct-to-mucosa anastomosis and preserved posterior layer. Close-up shows the pyramidal resection of pancreas, preserving posterior layer.
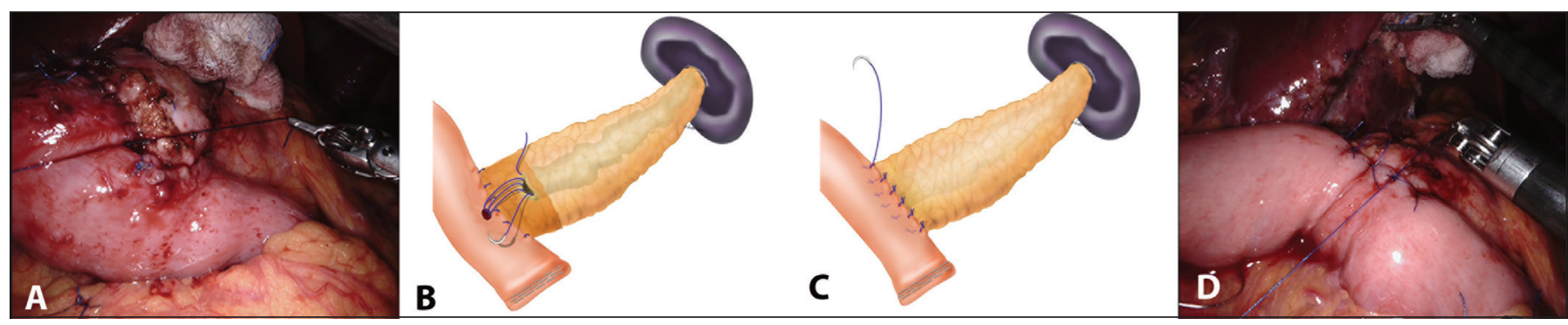

FIGURE 4. Alternative technique for redo pancreaticojejunostomy.

A. Intraoperative view after completion of duct-to-mucosa anastomosis. B. Schematic drawing shows duct-to-mucosa anastomosis. C. Schematic drawing after completion of redo pancreaticojejunostomy. D. Intraoperative view after completion of redo pancreaticojejunostomy.

cavity. Operative time for docking of the robotic system was four minutes. Redo pancreaticojejunostomy took one hour. Estimated blood loss was minimum, and recovery was uneventful. Patient was discharged on the 3 rd postoperative day. No pancreatic fistula was observed, and drain was removed on the 5 th postoperative day. Patient presented no recurrence of acute pancreatitis during one year of follow-up. Robotic redo pancreaticojejunostomy is feasible and safe. This alternative technique maintains this anastomosis assembled thus reducing the operative time and technical difficulties to perform this complex operation. This video shows the different steps necessary to perform this operation and will be useful for all surgeons having to perform a revision pancreaticojejunostomy.

\section{Authors' contribuition}

Machado MAC and Makdissi FF carried out the operative procedure. Machado MCC and Machado MAC conceived the technique. Ardengh JC and Makdissi FF supervised and commented on the manuscript. All authors discussed the results and contributed to the final manuscript.

\section{Orcid}

Marcel Autran C Machado: 0000-0002-4981-7607.

Fábio Ferrari Makdissi: 0000-0001-8202-5890.

Marcel C C Machado: 0000-0002-0729-1635

José Celso Ardengh: 0000-0002-5932-2499.

Machado MAC, Makdissi FF, Machado MCC, Ardengh JC. Pancreatojejunostomia revisional via robótica. Alternativa técnica no tratamento de estenose após duodenopancreatectomia. Arq Gastroenterol. 2020;57(2):221-2.

\section{REFERENCES}

1. Machado MA, Surjan R, Bassères T, Ardengh A, Makdissi F. Robotic pancreaticoduodenectomy after Roux-en-Y gastric bypass - Video article. Surg Oncol. 2019;29:118-9.

2. Sho M, Nakajima Y, Kanehiro H, Hisanaga M, Nishio K, Nagao M et al. A new evaluation of pancreatic function after pancreatoduodenectomy using secretin magnetic resonance cholangiopancreatography. Am J Surg. 1998;176:279-82.

3. Sledzianowski JF, Muscari F, Suc B, Fourtanier G. Recurrent pancreatitis after pancreaticoduodenectomy: reoperation for stenosis of the pancreaticojejunostomy. Ann Chir. 2004; 129:37-40.

4. Mucci-Hennekinne S, Brachet D, Clouston H, Pessaux P, Hamy A, Arnaud JP. Management of a stenotic pancreatico-digestive tract anastomosis following pancreatoduodenectomy. J Hepatobiliary Pancreat Surg. 2007;14:514-7.

\section{(cc) BY-NC}

